\title{
OVERVIEW OF REGULATORY INITIATIVES IN THE EUROPEAN UNION TO STIMULATE RESEARCH AND ACCELERATE ACCESS TO ORPHAN DRUGS AND OTHER HIGH MEDICAL NEED PRODUCTS
}

\author{
ALEKSANDRA BARAN-KOOIKER ${ }^{1 *}$, COEN KOOIKER ${ }^{2}$ and MARCIN CZECH ${ }^{3,4}$ \\ 'Department of Pharmacoeconomics, Faculty of Pharmacy, \\ Medical University of Warsaw, Żwirki i Wigury 81, 02-091 Warsaw, Poland \\ ${ }^{2}$ Independent research, The Netherlands \\ ${ }^{3}$ Department of Pharmacoeconomics, The Institute of Mother and Child, \\ Kasprzaka 17A, 01-211 Warsaw, Poland \\ ${ }^{4}$ Warsaw University of Technology Business School, \\ Koszykowa 79, 00-001 Warsaw, Poland
}

\begin{abstract}
The European Union (EU) is continuously working towards further promoting and facilitating registration and development of orphan medicinal products (OMPs). The goal of this article is to present a broad overview of existing legislation, programmes and incentives in the EU that allow for accelerated access of orphan drugs and high medical need products, as well as give some historical context and how these programs are interconnected. The authors conducted an explorative review of publicly available sources of regulatory and orphan drug policies as well as information on accelerated assessment programs in the EU. The authors identified and looked into the following programs and initiatives: orphan drug legislation, accelerated assessment, conditional approval, Priority Medicine (PRIME), compassionate use programs, off-label use and RTU, Medicines Adaptive Pathways to Patients (MAPPs), ADAPT-SMART, HTA initiatives (EUnetHTA, Parallel Consultation), STAMP, IMI and IMI2 and the European Framework Programmes (incl. FP7 IDEAL, Asterix and Inspire). It can be concluded that promoting innovation and early access to medicines has been firmly put on the agenda in the EU, with various programs and initiatives, either national or EU-wide. Hurdles in regulatory science remain present, especially for rare diseases, but fundamental research as done in FP7 could lead to more efficient orphan drug development. Barriers in the field of HTA, pricing and reimbursement still need to be addressed to enhance patient access. The true effectiveness of all these programs will only become clear over time.
\end{abstract}

Keywords: orphan drugs, regulatory pathways, PRIME, MAPPs, FP7

Despite being called 'rare diseases', the actual combined total prevalence in populations is believed to be between 6 to $8 \%$, due to the large number of rare diseases that have been identified (5000-8000) (1). Medicinal treatment for rare disorders (called 'orphan drugs' or 'orphan medicinal products' (OMPs)) is usually non-existent and new drugs are often difficult to develop: only about $1 \%$ of rare diseases have a treatment in the EU (2). In addition, many areas of the world are still lacking clearly defined regulatory processes or incentives for development of orphan medicinal products. The EU, Japan and the US have well-established regulatory policies to accelerate approval and access to OMPs (3-5) and recently China started catching up by introducing orphan drug legislation in $2017(6,7)$. Nevertheless, development of new orphan drugs is still sparse and patient access is often problematic due to pricing issues. The EU is continuously working towards further promoting and facilitating OMP research and development, registration and access. A good example is PRIME (Priority Medicines), an early and proactive program created by the European Medicines Agency (EMA) for drug developers to optimize generation of benefits and risks early in the development (8). Another major initiative is the Adaptive Pathway approach, which is intended to promote flexible use of the existing regulatory framework and tools, and to allow for an iterative regulatory assessment path and a staggered

* Corresponding author: e-mail: aleksandra.baran@gmail.com 
approval process, e.g. with expanding patient groups as more safety/efficacy data is generated (9). More than $30 \%$ of new medicines containing a novel active substance that were recommended for approval by the CHMP in 2016 used one or more of the different EMA tools for expedited review (10). A more fundamental approach to promote research is taken by the EU Framework Programmes for Research and Technological Development, such as Seventh Framework Programme (FP7) and Horizon $2020(11,12)$.

Several authors have presented overviews of expedited regulatory processes, such as Liu et al. who published an overview of orphan drug policies in the US, EU and Japan in 2010, in light of orphan legislation development in China (13). In 2017, the impact of Adaptive Pathways for payers was discussed by Vella Bonanno et al. (14). To the authors' knowledge, there is no recent publication that has discussed the range of new regulatory initiatives and developments for accelerated approvals in the EU. Therefore, the goal of this article is to present a broad overview of current initiatives, programs and incentives in the EU that are aimed at facilitating development, registration and early access to orphan drugs and other high medical need products. Some context is given and how these programs are interconnected and funded.

\section{METHODS}

The authors conducted an explorative review of publicly available sources of regulatory and orph- an drug policies as well as general information on accelerated assessment programs in the EU. Internet search engines and the search functions on specific websites were used to gather information and sources of new information using relevant keywords, including the EMA website, sites of national regulatory agencies (e.g. CBG-MEB, ANSM), the main website of the European Union and European Commission, the websites of IMI/IMI2, FP7 and Horizon 2020. The review was performed by 2 researchers. Information that was found was analyzed further to find references and links to other programs and relevant documentation, e.g. policy and legislative documentation, links to initiatives and developments on EU and the national level. New search keywords were added and adjusted when relevant new information was gathered. It is impossible to claim an exhaustive list of programs that have been compiled, however, the authors are confident all the major initiatives are captured within this article, as well as recent developments in the subject area. For research purposes, the authors also attended the 3-day EMA workshop on initiatives in Small Population Research methods projects and regulatory application, within FP7, in London March 2017.

\section{RESULTS}

The explorative search of the EMA website led to a large amount of information (policy documents, guidance, news items) on topics relevant to the chosen subject. The starting points were orphan drugs

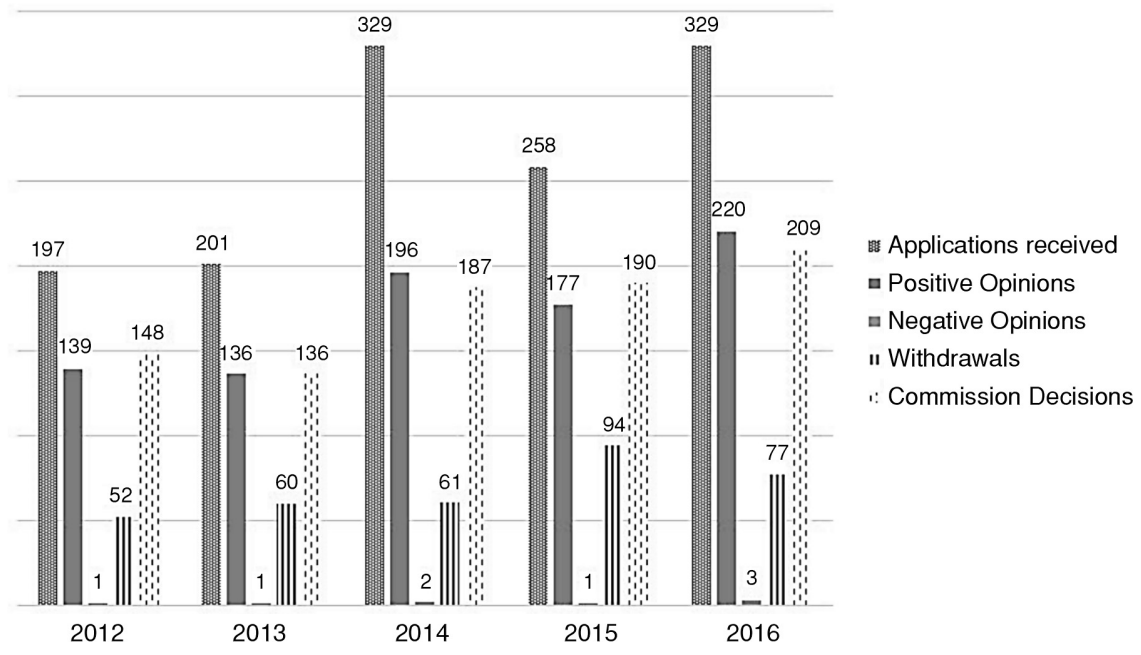

Figure 1. The number of orphan medicinal product designation applications (10) 


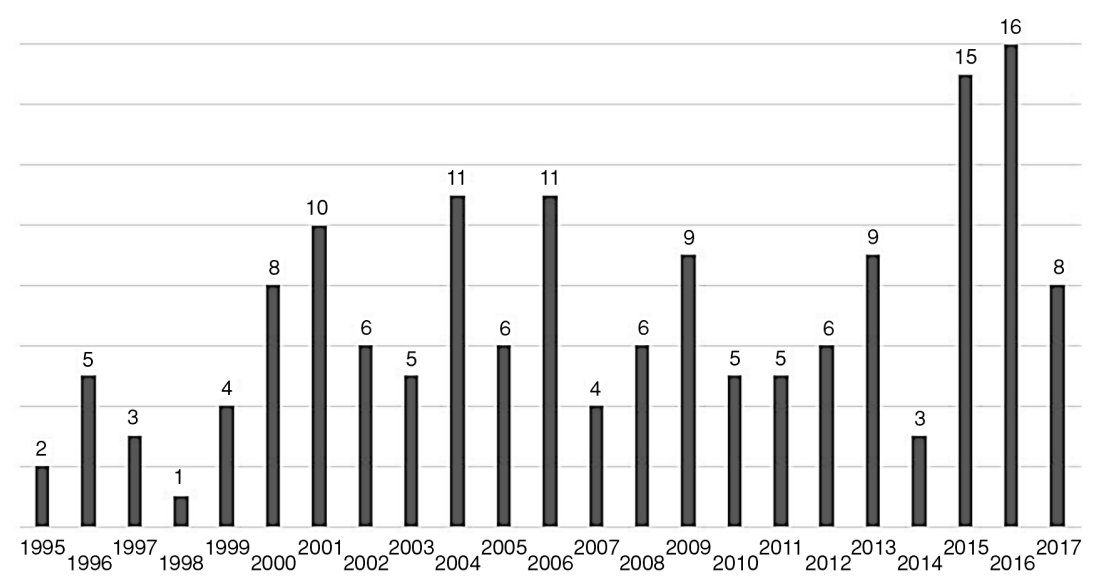

Figure 2. The number of medicinal products intended for rare diseases in Europe with European market authorization without orphan designation in Europe by date of EMA (10)

and orphan drug legislation. Further research focused on expedited access methods that the EMA has introduced, including accelerated assessment, conditional approval, approval under exceptional circumstances, PRIME, compassionate use programs, Medicines Adaptive Pathways to Patients (MAPPs), and parallel HTA consultation. Links to further information on STAMP, IMI and IMI2 (incl. ADAPT-SMART) and the European Framework Programmes FP7 and Horizon 2020 that were found and further reviewed. FP7 received special attention due to the 3 programs that focused on research in small population groups (IDEAL, Asterix and Inspire) that would be of relevance for rare diseases and orphan drug development, and the EMA FP7 workshop was attended. Data on off-label use programs (RTU) in France and Italy were found via internet search engines as well as via the ANSM and AIFA website. The results are structured in a similar fashion, with a focus first on orphan drugs and legislation, then a review of existing accelerated access initiatives, concluding with a section on the fundamental research programs that exist in the EU.

Introduction to the EU's orphan drug incentives

Having recognized that there are severe restrictions which limit manufacturers to develop drugs for rare diseases (mostly commercial and scientific limitations), the EU has adopted its Orphan Drug Regulation (15), which provides several advantages to OMP developers to stimulate research and increase the chance of successful approvals. The basic requirement for a drug to be eligible for these benefits is to have an Orphan Designation granted by the Committee for Orphan Medicinal Products (COMP) $(16,17)$ (for the number of OMP designa- tion applications please refer to Figure 1 and for the number of authorized OMPs to Figure 2). An orphan designation requires a justification of the rarity of the disorder (prevalence $<5 / 10.000$ ) and proof of lack of any effective current treatment, as well as data supporting potential clinically relevant outcomes (16). Designations are generally requested and granted during the development process (18). An already approved (non-orphan) drug can obtain an orphan designation for a new indication too, however, a new marketing authorization needs to be applied for in this case (with a new product name). Orphan and non-orphan indications cannot be part of the same marketing authorization in the EU (18). One of the main 'commercial' incentives offered to orphan drug developers is a 'market exclusivity' for newly approved orphan drugs, giving right to a 10year period where a similar competitive products cannot be placed on the market unless they would show a significant added benefit to patients, i.e. a clinically relevant advantage in efficacy, safety or a major contribution to patient care (15).

Orphan drug market exclusivity can be extended to 12 years if pediatric studies are performed with the drug via a pediatric investigation plan (PIP) (19). However, the exclusivity period can also be reduced to 6 years if sales of the orphan drug are sufficiently high at the end of the $5^{\text {th }}$ year to not justify the designation any longer (i.e. the product is sufficiently profitable) (15). In this case, the designation can be removed together with the market exclusivity (15). Around $60 \%$ of drugs with an orphan designation are intended for pediatric use (16). For non-orphan drugs, if the results of clinical studies from a PIP are included in the product information (even if study result are negative), the incentive is a patent exten- 
sion (supplementary protection certificate) by 6 months (20).

For drugs that are already authorized and have no patent protection and for which a new indication is investigated that would only target a pediatric population, a PUMA (Pediatric-Use Marketing Authorization) can be applied for. A PUMA grants 10 -year 'market protection', meaning that generic products are allowed to be registered, but not placed on the market (as opposed to the more common 'data protection' rules, which prevent generic registration). Market protection could be applicable for orphan pediatric indications for existing drugs (21). For the number of designated orphan medicines for the treatment of children and adults (2012-2016), please refer to Figure 3.
'Protocol Assistance' is a form of Scientific Advice provided by the EMA aimed specifically at optimizing the clinical development for orphan drugs and to provide assistance on how to meet regulatory requirements (22). Orphan drugs automatically gain access to the Centralized Procedure (CP) to be able to gain simultaneous approval in all EU member states. In fact, since 2004 it is no longer possible to use any other regulatory route than the $\mathrm{CP}$ to register a designated orphan medicinal product (23).

Another incentive of financial nature for all company sizes is a reduction of orphan drug-related regulatory fees, which are $-100 \%$ on pediatric related protocol assistance and pre-authorization GMP/GCP-inspections. Micro and small/medium

Table 1. Overview of fee reductions for orphan drug manufacturers and SME's (10).

\begin{tabular}{|c|c|c|}
\hline & Small/medium enterprises (SME) & Large enterprises \\
\hline Orphan drug-related incentives & & - \\
\hline Protocol Assistance - pediatric & $100 \%$ reduction & $75 \%$ reduction \\
\hline Protocol Assistance - non-pediatric & $100 \%$ reduction & $100 \%$ reduction \\
\hline Pre-authorization GMP- and GCP-inspection & $100 \%$ reduction & $10 \%$ reduction \\
\hline Orphan drug marketing application & $100 \%$ reduction & - \\
\hline Post-authorization activities in the first year & $100 \%$ reduction & - \\
\hline Non-orphan related incentives (SME legislation) & & - \\
\hline EMA Scientific Services & $90 \%$ reduction & \\
\hline Post-approval inspections & & \\
\hline
\end{tabular}

Designated orphan medicines for the treatment of children and adults (2012 - 2016)

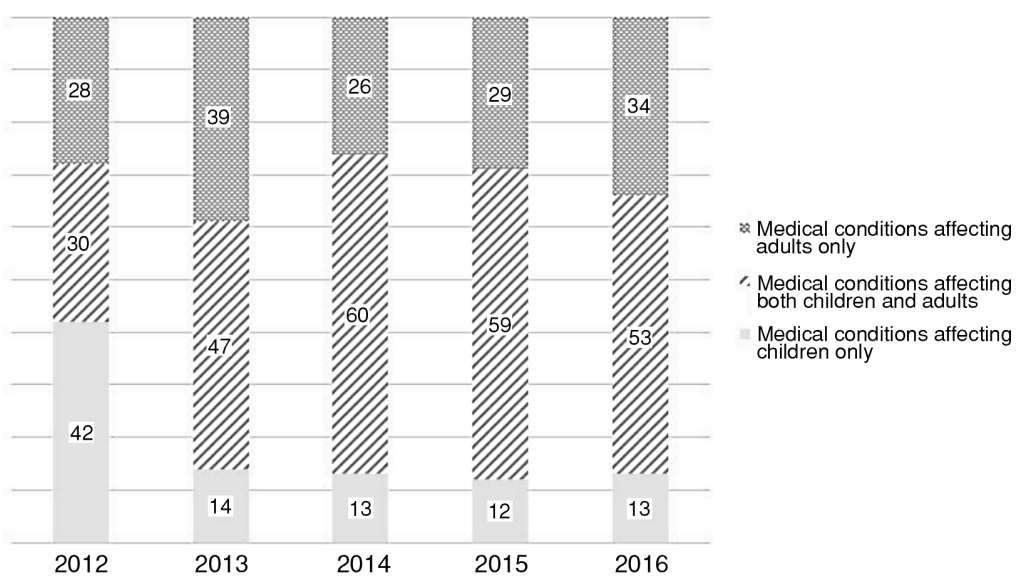

Figure 3 The number of designated orphan medicines for the treatment of children and adults (2012-2016) (10) 


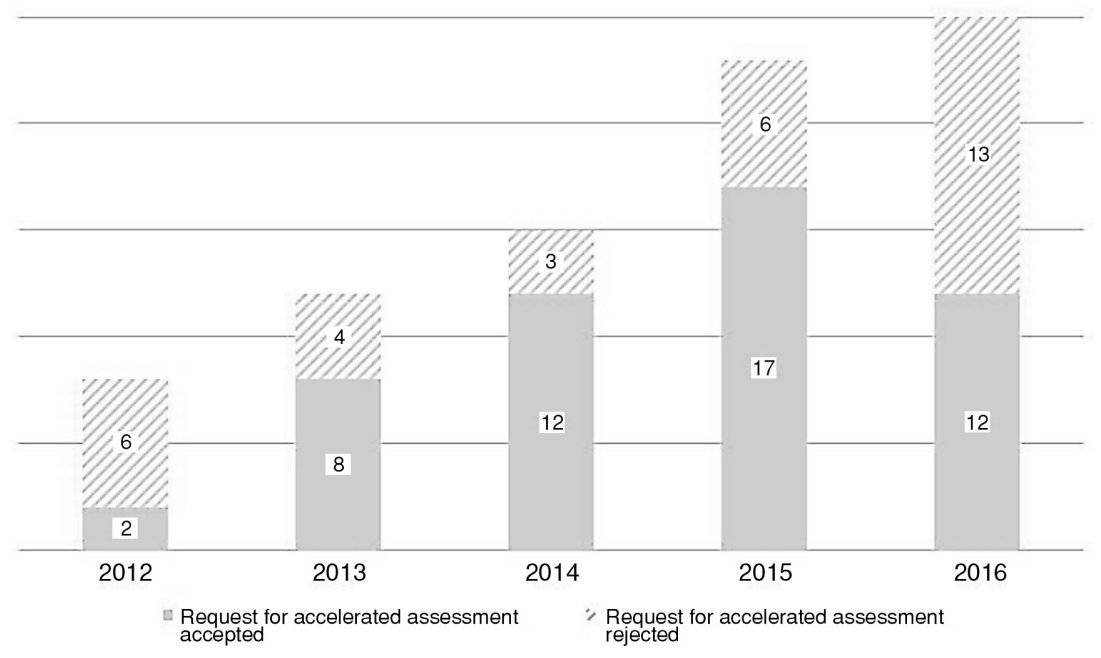

Figure 4. The number of accelerated assessment requests (2012-2016) (10)

enterprises (SME) further enjoy a $100 \%$ fee reduction on orphan drug marketing authorization applications (10\% for bigger companies), $100 \%$ reduction on post-authorization activity fees in the first year and a $100 \%$ reduction on non-pediatric protocol assistance (75\% for bigger companies). The SME legislation 2049/2005 provides a further $90 \%$ fee reduction on post-approval inspections and $100 \%$ on EMA Scientific Services, exclusively to SME's (24). For an overview of relevant fee reductions, see Table 1 .

Health authorities recommend drug manufacturers to apply for an orphan designation simultaneously in the EU, US and Japan. However, as the criteria are not (yet) internationally harmonized the assessments are still done independently. As a result, the EU does not legally recognize an orphan status granted in any other region (25).

Initiatives aiming at facilitating the development and assessment of innovative products

The EU has identified that there were major commercial, legislative and scientific hurdles for drug manufacturers to develop drugs in high medical need areas, especially in small population groups. Several EU-wide programs have been created to facilitate development and registration of new molecules, e.g. by making regulatory processes more flexible and straightforward, investigating new statistical methodology, creating innovative trial designs, as well as initiatives in the HTA arena. An overview of these initiatives is presented below.

\section{Accelerated assessment}

To accelerate access to drugs that could target an unmet medical need, both orphan and non-orphan drugs can apply for an accelerated assessment procedure with the EMA that was adopted in 2006 (26), which reduces the regulatory assessment period to 150 days (instead of 210, excluding clock-stops). In order to qualify for accelerated assessment, the drug has to be a therapeutic innovation and of "major public health interest", which should be justified by the applicant. Drugs with an orphan designation are not automatically qualified for accelerated assessment as it still needs to be established if it is intended to meet a 'major public health need' (27).

During 2012-2016, 83 requests for accelerated assessment were made to the EMA, of which 51 were accepted (10) (for the number of accelerated assessment requests please refer to Figure 4).

\section{Conditional approval / exceptional circumstances}

Next to accelerated assessment, another program exists that can expedite the regulatory approval process, called conditional approval, which can be granted based on less comprehensive data than normal/full approvals, in cases when the immediate availability of the medicine outweighs the lack of available evidence. The benefit/risk bal- 
ance still should be positive, the drug has to address an unmet medical need and, importantly, it must be likely that additional data becomes available (28). Special post-authorization obligations, such as the collection of pharmacovigilance data after approval, may be a requirement. Conditional approvals are valid for one year and need to be renewed yearly, until either a full approval is granted or until the conditions are no longer met (and the marketing authorization is withdrawn). Between 2006 and 2016, 30 medicines were granted conditional approval by the EMA (28).

A marketing authorization can also be granted under "Exceptional Circumstances", which is similar to a conditional approval, with the exception that it is unlikely that further supporting evidence can be generated for the drug because either the disease is too rare, scientific knowledge is inadequate or it would be unethical to perform a study (28). This marketing authorization can be subject to specific obligations, such as being limited to an Rx prescription status, the requirement for strict medical supervision during use (i.e. hospital only) or the need to conduct additional studies (to re-assess the benefit/risk balance). The product information has to clearly reflect the fact that scientific information concerning the medicine is still inadequate. By definition, (ultra-)orphan drugs are the most likely candidates to receive approval under exceptional circumstances (29).

\section{PRIME}

An expansion of EMA's existing expedited review programs (e.g. accelerated assessment) has been implemented in the PRIME initiative (PRIority MEdicines), which was launched in 2016. PRIME is a program targeted towards developers of promising drugs that address an unmet medical need, to improve the generation of robust data on benefits and risks already in an early phase of the development. The enhanced and early dialogue with EMA within PRIME is aimed to optimize development plans, clinical trial and protocol designs, regulatory strategies and to speed up the evaluation of an eventual marketing authorization application. Products eligible for PRIME can make use of the existing tools, and it can be expected that such a product will be granted an accelerated assessment during the marketing authorization application $(30,31) .29$ products were granted PRIME until October 2017 (32).

\section{Compassionate use programs}

Whenever a drug is under development (or registered elsewhere) and an urgent medical need has been identified that this drug could address, i.e. when there is no effective treatment available to patients suffering from a serious or life-threatening condition (and clinical trials are excluded for these patients), many EU countries allow early access via a 'compassionate use program' (CUP) (33). In essence, a CUP allows for the treatment of a cohort of patients with unregistered drugs outside the setting of a clinical trial. If such a request is based on a single patient, such a program would be called a 'named patient program (NPP) or 'individual patient program'. A CUP would generally last until the drug has been granted a marketing authorization or until patient access has been achieved (i.e. reimbursement), depending on local guidelines. The costs of a CUP or NPP are generally borne by the drug developer.

Most medicines that are distributed via a compassionate use program are processed by local initiatives and national CUP legislation, and a 2016 publication showed that 20 out of 28 EU member states had a clearly established CUP framework (except Cyprus, Estonia, Greece, Ireland, Lithuania, Luxembourg, Poland, and Slovakia) (34). A CUP can also be granted by the EMA (CHMP) on an EUwide level and up till 2017 five drugs were granted a CUP by the CHMP, all targeted towards viral or pandemic conditions: hepatitis $\mathrm{C}$, influenza and pandemic H1N1 flu (sofosbuvir, ledipasvir/sofosbuvir, daclatasvir, zanamivir IV, oseltamivir IV) (30).

Notably, Italy has a compassionate use law (35) that has been broadened to drugs for which therapeutic alternatives do exist (36). This has been challenged by pharmaceutical manufacturers. For more information, see also the "Off-label Use" section below.

\section{Off-label use / RTU}

The use of medication for an indication that is not approved, i.e. off-label use, is known to be widespread. A recent EU report indicates that $7-95 \%$ of medicine prescriptions are off-label (inpatient setting) (37). Rare diseases are often considered to be prone to treatment with off-label medication, due to disease severity, the limited number of approved treatments, and the high percentage of pediatric patients, although exact figures are lacking.

Companies are not allowed to promote a drug commercially for off-label indications, due to obvious concerns about safety and efficacy, and off-label use is therefore usually not reimbursed. Off-label use is often done without adequate monitoring, which does not improve the lack of information on the benefit-risk balance in specific indications. 
France has created a unique legal framework to enable the prescription of already registered and commercialized drugs for off-label use, i.e. for nonauthorized indications, called Recommendation for Temporary Use or RTU. Since its inception, 16 products have been allowed for temporary off-label use in France (38). France also has compassionate use and a named-patient program, under the socalled Temporary Authorisation for Use initiative, or ATU.

An RTU is possible under strict conditions, being that there is an unmet therapeutic need (e.g. no available alternative with the same active ingredient, dose and pharmaceutical form) and the benefit/risk ratio is assumed to be favorable, based on available scientific data on efficacy and safety (39). An RTU can only be initiated by the regulatory agency ANSM after off-label use has been identified in clinical practice. When RTU was introduced in 2012, a requirement existed that there should be no therapeutic alternative on the market. In 2013, the ruling was changed to allow for an RTU if it would avoid a significant financial impact on the healthcare budget (40). After that, bevacizumab started to be used off-label in an RTU for wet macular degeneration (WMD), which was challenged by the manufacturers of competing products with a registered WMD indications (ranibizumab, aflibercept). A court ruling by the European Court of Justice (ECJ case Novartis Pharma GmbH v Apozyt GmbH (Case C-535/11)) has allowed this practice in 2014, i.e. an RTU is allowed even when an alternative product (with a different active substance, dosage and pharmaceutical form) is available on the market, leading to a change in RTU requirements in 2014 (41). The RTU was challenged by the manufacturers, but the French Supreme Court upheld the RTU in February 2017 (Conseil d'État N 392459 - February 24, 2017).

An RTU comes with the obligation for a drug manufacturer to collect real-world data on the new indication, described in a patient monitoring protocol (39). This data might support an official indication extension request later on, but results from clinical trials will still be required an indication extension. When ANSM plans to issue an RTU, the manufacturer must submit all relevant information for the use of the product in the unlicensed indication, such clinical/non-clinical data, a list of regulatory approvals elsewhere and information on planned and ongoing trials for this indication. For rare diseases, the ANSM will consult with the reference center (if existing) or the national cancer institute INCA. Based on the presumed benefit and expected risks the ANSM will then make a decision on granting the RTU. If approved, the RTU will be published and implemented. A single RTU can be applied to multiple products (with a similar mode of action) and can last up to 3 years, but it can be renewed. Pharmacists and physicians are obliged to participate in data collection (39). It is not allowed to advertise the product for the RTU indication and medical prescriptions need to be clearly identifiable as off-label use.

\section{Italy}

Italy planned to introduce an update to their compassionate laws by the end of 2017, which will change the definition of compassionate use to include 1. off-label use of licensed products (which is contradictory to the EU definition (42) and 2. the on-label use of already licensed products which are not available in Italy (i.e. in situations where reimbursement is still awaited). It remains unclear whether manufacturers can be reimbursed for the medicine supply in a CUP (as allowed under Italy's "Di Bella" law 94/1998) (43) or whether medicines will have to be supplied for free. The regulatory body AIFA keeps the decision rights to allow off-label treatment and reimbursement of selected drugs.

\section{Medicines Adaptive Pathways to Patients (MAPPs)}

Adaptive pathways are a new and broader scientific concept that makes use of the existing EU regulatory framework, with the goal to improve medicine development and the creation of data that would enable progressive and early access to (novel) medicines for well-defined patient populations with a high unmet medical need (9). One of the key drivers for the development adaptive pathways is the realization that conventional randomized clinical trial (RCT) designs are not fit for many diseases, and new methods are needed to develop new approaches to data generation without a decline in the quality of evidence. Adaptive pathways are centered on iterative development (incl. expanding patient populations during development, early benefit-risk assessment), the use of real-life data in addition to clinical outcomes and early discussions with patient organizations and HTA bodies and using early access methods. Existing regulatory tools are intended to be used, such as scientific advice, compassionate use, conditional approval and utilizing observational data collected from pharmacovigilance sources and patient registries. MAPPs can be used to manage risks in situations of limited available evidence. To be eligible for MAPP, treatments 
need to be in the area of high medical need where traditional evidence generation is difficult and larger clinical trials would not be feasible or justified from a benefit/risk viewpoint. The EMA ran a pilot on MAPPs from 2014 to 2016, which led to the (draft) proposal to increase the number of (pre-submission) meetings with the EMA during the development of eligible drugs. A follow-up to the pilot ran until the end of 2016 and final conclusions and further recommendations are awaited. EMA received 62 applications and selected 18 proposals for face-to-face meetings. At the end of the pilot, 6 of the applicants had received parallel advice from EMA and HTA bodies and 1 benefited from EMA scientific advice (44).

\section{HTA initiatives - parallel consultation (Joint HTA-Scientific Advice)}

As reimbursement has become a major hurdle in patient access in recent years, EMA has sought collaboration with HTA agencies to provide companies with dual support. This has resulted in the "Parallel Consultation" initiative (45) which is combined scientific advice addressing both scientific/regulatory requirement and HTA requirements, supported by the EMA and the European European Network for Health Technology Assessment (EUnetHTA). EUnetHTA was initiated in 2006 and is comprised of 78 HTA organizations from 29 countries (46). In recent years, EUnetHTA has had 3 major activities (work packages) (47): Joint Action 1 (JA1, 2010-2012) focused on bringing European HTA agencies together and finding ways to harmonize and exchange information and improve relative effectiveness assessments, JA2 (2012-2015) focused on the tools for cross-border HTA collaboration and further harmonization, and JA3 (2016-2020) is focused on scientific and technical collaboration between HTA bodies and to prepare for a fully functional stand-alone network by 2020. Joint Action 2 included the first collaboration efforts with EMA and a joint 2012-2015 work plan, resulting in the 'parallel consultation' initiative in 2017. EMA and EUnetHTA announced a 3-year work plan in 2017, running until 2020 (47).

A similar project, specifically focused on rare diseases, is the European MoCA project (Mechanism of Coordinated Access to Orphan Medicinal Products), which is a step towards international harmonization and improvement of patient access to orphan drugs in the EU. This platform, created in 2010, aims to facilitate an early dialogue on pricing and reimbursement during the development phase of OMP's between pharmaceutical companies and competent authorities (48).

\section{STAMP}

In addition to incentives offered by the EU and EMA, individual member states also provide benefits within the boundaries of their national legislation to promote development and access to orphan drugs, in the form of research grants, early access programs, fee waivers/reductions and reimbursement related benefits. The EU Commission has recognized that the plethora of national initiatives are worth to be looked at by the EU, and created the STAMP expert group (Safe and Timely Access to Medicine for Patients) in 2015 for this purpose (49). The members are representatives of regulatory bodies, healthcare ministries, national assessment and approval agencies, and the EMA. The goal of STAMP is to exchange information on national programs and explore methods to use existing EU regulatory legislation in more effective ways and to find methods to speed up access to innovative medicines. Since its conception, STAMP has provided an interactive communication platform for stakeholders and discussed a range of access-related topics. STAMP has been a participant in the development of the PRIME scheme and it is further discussing the "repurposing" of existing medicines, off-label use, and early access tools, among others (50).

\section{IMI \& IMI2}

The EU Joint Technology Initiative on Innovative Medicines (short: Innovative Medicines Initiative or IMI) is a public-private partnership focused on health between pharmaceutical companies, universities, SMEs and patient organizations, with the EMA as a scientific leader. IMI's goal is to "significantly improve the efficiency and effectiveness of the drug development process with the longterm aim that the pharmaceutical sector produces more effective and safer innovative medicines". IMI ran from 2008 to 2013 and resulted in a list of 59 relevant projects and 1134 publications (51).

Due to the deemed success of this large-scale industry-EU partnership, IMI2 has been implemented, which will run from 2014-2024 (49). IMI2's "Strategic Research Agenda" is aligned with the EU's "Health Research Priorities" and on a higher level with "Horizon 2020 (FP8)" (discussed below) (52). IMI2 intends to improve the current drug development process, by promoting of new scientific tools and biomarkers, by reducing the time to reach clinical proof of concept, by increasing the success rate in clinical trials of priority medicines 
and by developing high unmet need treatments (e.g. for Alzheimer's) (53).

One of the results of IMI2 is the creation and funding of the ADAPT-SMART consortium, which comprises of all relevant stakeholders in the healthcare system: patients, academia, industry, regulators and HTA bodies, with the objective to establish a platform for dialogue on (improving) MAPP implementation by the EMA and industry (54). Rare disease patient representatives are also included, such as EURORDIS.

ADAPT-SMART (Accelerated Development of Appropriate Patient Therapies: a Sustainable, Multistakeholder Approach from Research to Treatmentoutcomes) launched September 2015 and is planned to run for 30 months, i.e. early $2018(55,56)$. Goals of ADAPT-SMART include identifying hurdles in the proposed adaptive pathways and getting multistakeholder input to resolve these, creating common definitions and best practices, and finding ways to incorporate new initiatives (coming from other EU programs, e.g. IMI, FP7) into MAPPs. Key deliverables from the program will be finding new sources of evidence and the development of new tools for the evidence generation that would be required and suitable in MAPPs. Results are expected in 2018.

\section{EU Framework Programme 7 - New methodolo- gy for Small Population Groups}

The Framework Programmes for Research and Technological Development (FP1 to FP7) are funding programs to stimulate and support research done in the EU. FP6 ran from 2002 to 2006 and included several projects aimed at rare diseases, such as EUROWILSON which looked at how to achieve sufficient patient numbers by linking phase 1 and 2 trials (57). FP7, with a total budget of 55B over 2007-2013, led to the sponsoring of 25.000 research related projects $(58,59)$.

One of the programs within the "Health" section of FP7 built on the realization that there is an "unmet scientific need" in the area of trial design and (statistical) methodology in the rare disease arena, and was focused on „New methodologies for clinical trials for small population groups" (SPG) (60). Rare diseases are characterized by small patient populations, but the average number of enrolled patients in clinical trials still is sufficient: on average 761 patients (median 538) vs. 3549 in non-orphan drug trials (median 1588) (61). However, rare disease heterogeneity and a large percentage of pediatric patients make proper trial setup difficult. When conventional methodology is used that is most suited for large randomized clinical tri- als, it is often difficult to generate valid outcomes necessary for marketing authorization. Further complicating factors are the lack of tailored guidance on clinical development and "knowledge models" to help disease understanding and predict treatment outcomes, among others.

Three public-private projects were initiated within FP7 to look specifically at ways to improve or develop a new methodology for design and analysis of clinical trials in small populations: IDEAL, InSPiRe and ASTERIX. The 3 projects had a total combined budget of $10.7 \mathrm{M}$ (largely sponsored by the EU). Each looked at different subject areas, although the investigated and proposed concepts often overlapped. The last project ended in September 2017 (InSPiRe). A large number of recommendations, scientific publications, presentations, webinars, and conferences were the main result of these projects, as well as improved or newly developed statistical software packages. FP7 has been followed up by Horizon 2020, which is running from 2014 to 2020.

\section{FP7: IDEAL - Integrated DEsign and AnaLysis of small population group trials}

The IDEAL project group looked at possible improvements of statistical design and analysis methods for SPG trials, to enable "efficient assessment of the safety and/or efficacy of a treatment". The focus was laid on randomization, extrapolation of dose-response data, adaptive trial design, mixed model design, pharmacokinetic and individualized designs, trial simulation, genetic factors, decisiontheoretic considerations, and biomarkers/surrogate endpoints. IDEAL was comprised of researchers from European universities, research institutes and industry. The project's 11 work packages resulted in 63 scientific publications, 33 recommendations, new statistical software packages and program code (e.g. a patient randomization package) (62). Special attention was paid to 'within-patient trials'. These $n=1$ designs are randomized trials where individual patients are randomized in repeated cycles to both treatment and control (e.g. placebo) with a washout period in between cycles. The advantages of these trials are that patients are their own controls and more than one measurement of effect is generated, leading to increased efficiency. Despite obvious limitations, i.e. $n=1$ trials are suitable only for chronic diseases where a washout period would not lead to unacceptable safety concerns, they are by definition applicable to small population research. Several recommendations around the design and analysis of $\mathrm{N}$ of- 1 trials were published by the IDEAL group. 
FP7: InSPiRe - Innovative methodology for small populations research

The main goal of InSPiRe was to define new methods for SPG research, where evaluation of healthcare interventions is often infeasible or impossible. InSPiRe was comprised of experts in innovative clinical trial design from industry, academia, and regulatory agencies, and it researched clinical trial and data analysis methods. The focus was laid on optimizing trial designs, to enable efficient and statically rigorous data collection from a limited number of patients and how to identify subpopulations of patients where maximal effectiveness of the treatment can be expected. Four specific subject areas were selected: early phase dose-finding and how to better incorporate PK/PD data, while limiting the required sample-size; decision-theoretic trial design methods evaluating the required level of trial evidence needed for clinical practice (please refer to Figure 5); confirmatory trials and personalized medicine, taking into account genetic factors and biomarkers; use of evidence synthesis in the planning and interpretation of clinical trials, to compensate for sparseness of data. Incorporating data other than primary clinical endpoints into these new designs, such as data from registries or other trials, was analyzed as well. Varying the required level of evidence depending on the population size, and health economic aspects of the trial design were also investigated. Adaptive enrichment trials, 2-stage trials which allow for an adjustment of the investigated patient population after interim analysis (e.g. based on biomarker data), were discussed. InSPiRE led to over 21 publications (excluding 10 still under review) in healthcare policy, medical and methodological journals. Publication in methodological journals often a prerequisite for the use of new methods in clinical development and acceptance by regula- tors. InSPiRe members were directly involved in the EMA Reflection paper on extrapolation of efficacy and safety in pediatric medicine development, which in turn might influence ICH guidelines globally (63-65).

\section{FP7: ASTERIX (Advances in Small Trials dEsign for Regulatory Innovation and eXcellence) \\ ASTERIX was a joint project run by academia,} clinicians, patient organizations and industry statisticians, collaborating together on the development of more reliable, cost-efficient and effective research designs for rare diseases, with a high level of patient centricity. Its main objectives were:

- Developing design and analysis methods for single trials and series of trials in SPG;

- Including patient-level information/perspective in trial design and decision making;

- Validating new methods and propose improvements for regulatory purposes.

An overall goal was to improve the statistical power of SPG trials, by combining data from various sources. A secondary goal was to find ways to involve patients in trial design and how to better inform them, before and during trials, resulting in improved recruitment and decreased dropouts. One of the results of ASTERIX is the clustering of rare diseases into 6 groups, based on disease characteristics and optimal design choices (data gathered from $100+$ regulatory assessment reports), which can help further methodology improvement and regulatory assessment. A method was developed to include patient preferences into (the weighing of) disease outcomes. Goal Attainment Scaling (GAS) was investigated, which is a method to measure disease outcomes when generic outcome measures are not suitable and development and validation of diseasespecific outcome measures is problematic, i.e. in

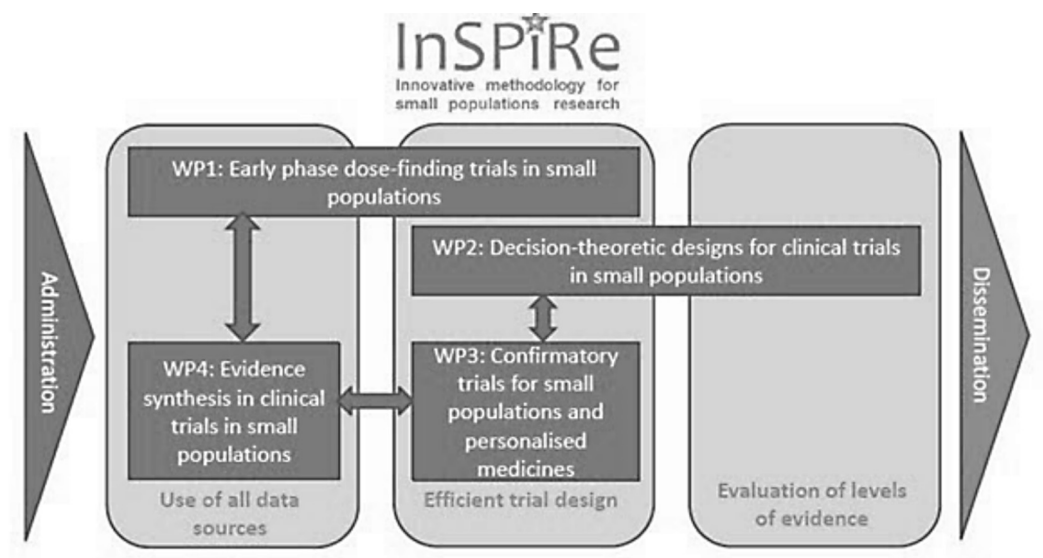

Figure 5. InSPiRe (65) 
case of disease heterogeneity (66). Based on the individual patient's preferences, clinical outcome goals are defined for each patient and weights are assigned to each different goal. After treatment, an assessment will be made as to what level of each patient selected goal has been reached. This makes GAS a potential instrument for outcome measurement in (heterogeneous, chronic) rare diseases, as well as a method to compare the effectiveness of different interventions. GAS has been described by Kiresuk and Sherman in 1968 (67), but it is getting renewed attention lately in the context of rare diseases (68).

\section{DISCUSSION}

The topic of this publication is in constant movement, making it hard to capture all information and keeping it up-to-date with the latest changes. E.g. during the creation of this article, several guidelines have been updated, projects progressed or finalized, and new legislation was announced or implemented. In contrast to the EMA, which publishes all its initiatives on its website instantly, national initiatives are often less easy to find and are usually drafted in the local language. Even though the authors attempted to create a complete and current overview, this may have led to missing certain recent important national developments. The same applies to orphan drugs: officially there are 133 drugs with an orphan designation registered in the EU (10), but many medicinal products were registered nationally before the EMA and Orphan Drug legislation were introduced. This makes it difficult to produce a clear overview of the total European orphan drug market or to compare the EU with the USA, which introduced its orphan drug law already in 1983 (69).

More than $30 \%$ of new medicines containing a novel active substance that were recommended for approval by the CHMP in 2016 used one or more of the different EMA tools for expedited review, which shows uptake is significant (10). It is likely that manufacturers of promising products will make use of several incentive programs, e.g. an orphan designation first, a MAPP early in the development and use accelerated assessment afterward on the way to regulatory approval. However, uptake is still on the low side compared to the US where $60 \%$ of approved drugs used one or more expedited pathways in 2015 (70). These numbers may reflect a delay in patient access in the EU. Relevant examples of this are nivolumab and pembrolizumab, two major breakthroughs in immuno-oncology. Both products were granted FDA approval in July/September 2014 respectively, but only by July 2015 in the EU (71). Even though FDA's expedited pathways may not be identical, the reason for this difference remains unclear. Familiarity of drug manufacturers with these programs may play a role, as well as the preference to choose the US for first drug approval.

If the common intent is to accelerate access to orphan drugs even further, it can be argued that these products should be automatically qualified for EMA expedited programs, which is not the case presently. On the other hand, accelerated access only shaves 2 months of the whole drug development timeline of $10+$ years, so a more holistic approach to speed up the whole clinical development, such as the MAPP, PRIME, and initiatives to promote fundamental science and clinical trial methodology (as in FP7), can be valuable. Given the rarity of rare diseases and the general sparseness of data, a combined assessment of orphan designations and/or marketing authorizations of orphan drugs by FDA, EMA, and other agencies could be considered. Medical experts in rare diseases already work closely together in international networks, it seems logical that regulatory agencies would follow suit. Harmonization of intellectual property rights (e.g. patents) would be beneficial but is not to be expected soon.

Having early discussions with health authorities can have positive impact on clinical development timelines: the FDA estimated a median clinical development time of 9.0 years (all new molecular entities) and 10.2 (orphan drugs) for companies who did not seek early advice, vs. 6.5 and 6.7 years respectively for companies that did (72). It remains to be seen what the effective benefit of EU's accelerated access programs will be in terms of time to reach regulatory approval, reimbursement or patient access in general.

Compassionate use programs can be done on a European level via the CHMP, but this route is hardly utilized. The majority of compassionate use instances are done via requests with the national competent authority, via local legislation, which are also less transparent for this reason. The rationale for not using EU-wide CUP is not clear, but it can be argued that the industry has commercial arguments (CUP costs are borne by the manufacturer and revenues could be reduced after approval), medical reasons (only limited patient numbers in countries) and legal argumentation (hesitation to promote nonapproved drugs/off-label use, difficulties with adequate physician training or the collection of adverse events, for example). Broad compassionate use pro- 


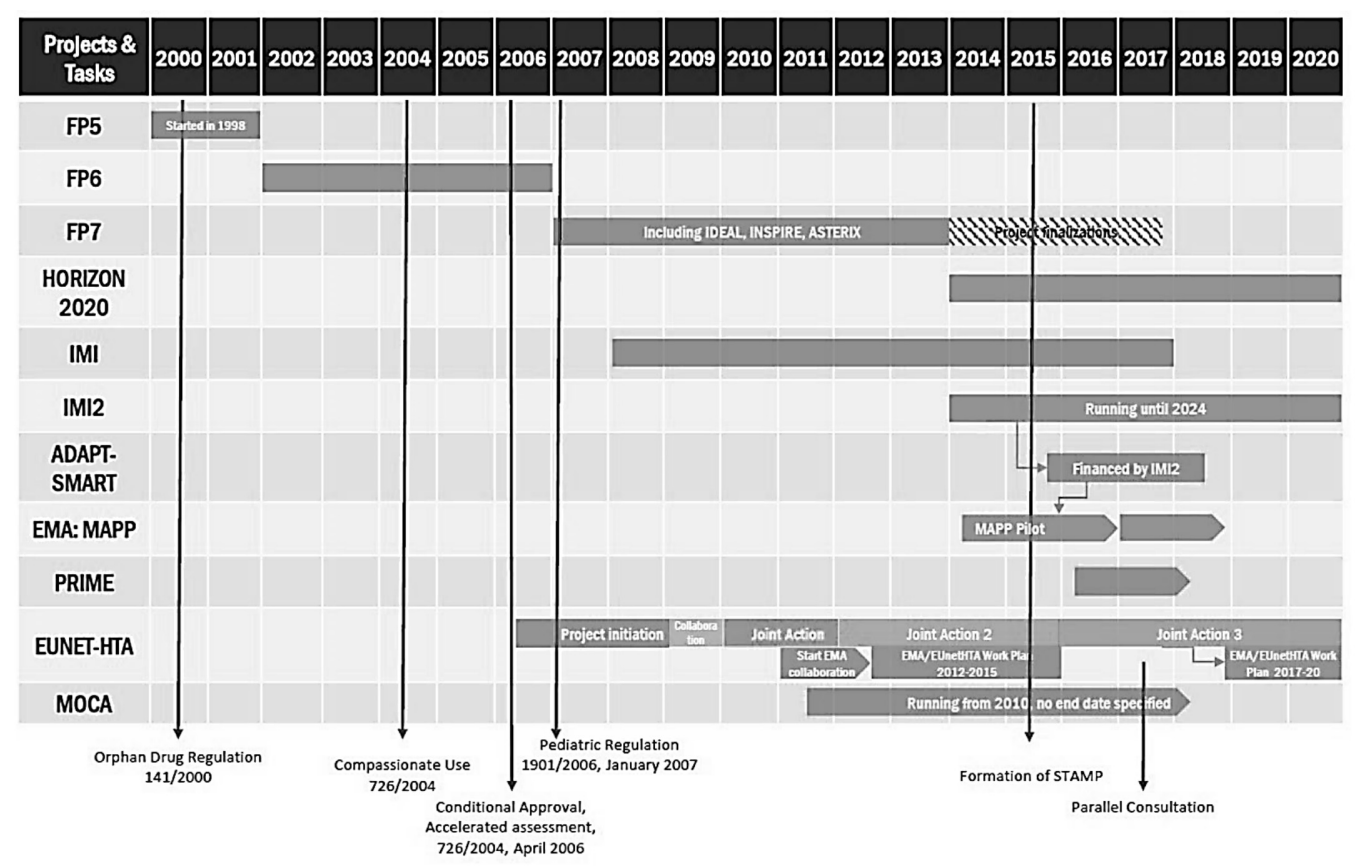

Figure 6. Overview of presented in this article EU initiatives by year

grams could also compete with patient recruitment of running clinical trials, which can be a relatively large problem in rare diseases. If during drug development (serious) adverse events would occur in patients in a CUP who would normally be excluded from clinical trials, this could endanger the whole development program of the drug itself. Identifying and managing these risks together with the industry could be helpful in promoting the use of CUPs.

Several countries have official programs for off-label use (France, Italy), but most countries have a less clearly established framework. The French and Italian initiatives indicate that there is a need for an expanded definition of CUP (i.e. new indication for existing products), as well as for managed offlabel use. Having a proper legal framework for offlabel use could increase data on drug effectiveness and reduce the risks involved. However, it is to be expected that discussion between industry and regulators will continue on the legal aspects.

As this publication has shown, a lot of initiatives and harmonization exist within the regulatory field. However, harmonized HTA approaches have not yet matured, leading to inequality in access to (reimbursed) OMPs in Europe. EUnetHTA is a good initiative, however, a lack of tangible results after 11 years of development can be clearly identified. The effects and uptake of the new parallel HTA/EMA consultation will become visible in the coming years. ,The recently announced EU HTA regulation will likely build on previous initiatives and be a significant step towards harmonization HTA process across the EU',(73).

\section{CONCLUSION}

Disease progression and permanent medical complications of rare diseases can already occur at a young age. Thirty percent of rare disease patients die before the age of five (73). This makes early access to treatment particularly important for rare disorders, to either cure or prevent disease progression. Expedited pathway initiatives are necessary steps in accelerating development and approval timelines for orphan drugs and other high medical need products. Especially for rare diseases, where patient populations are small and orphan drug development is often done globally, further international collaboration would be beneficial.

It can be concluded that since 2000, promoting early access to (innovative) medicines has been firmly put on the agenda in the EU, resulting in various programs and initiatives, either national or EUwide. The Orphan Drug Legislation has created clear and practical guidance for both drug manufacturers and regulators, facilitating the registration of new orphan drugs resulting in a growing number of 
orphan drug approvals. Overarching frameworks such as the framework programmes FP7, Horizon 2020 and IMI and IMI2, have led to multi-stakeholder research into how to address hurdles in medicine development, both from policy and regulatory perspectives, as well as with novel scientific methodology and fundamental research.

The overall effectiveness of all these programs will be shown by the number of (orphan) drugs registered and a reduction of time to market.

\section{REFERENCES}

1. https://www.eurordis.org/content/what-raredisease (26. 11. 2017).

2. European Commission: Inventory of Union and Member State incentives to support research into, and the development and availability of, orphan medicinal products, STATE OF PLAY 2015. SWD(2015) 13 FINAL, SANTE Brochure 2101 , Health and Food Safety https://ec. europa.eu/ health//sites/health/files/files/orphanmp/doc/or phan inv report 20160126.pdf (26.11.2017).

3. http://www.ema.europa.eu/ema/ (26. 11. 2017).

4. https://www.pmda.go.jp/english/ (26. 11. 2017).

5. https://www.fda.gov/ (26. 11. 2017).

6. CFDA Notice No. 52/2017 http://www.sda.gov. cn/WS01/CL0778/172567.html (26. 11. 2017).

7. Cheng A., Xie Z.: Orphanet J. Rare Dis. 12, 13 (2017).

8. http://www.ema.europa.eu/ema/index.jsp? curl=pages/regulation/general/general content 000660.jsp (26. 11. 2017).

9. http://www.ema.europa.eu/ema/index.jsp? curl=pages/regulation/general/general content 000601.jsp (03. 12. 2017).

10. European Medicines Agency: EMA Annual Report 2016 http://www.ema.europa.eu/docs/en GB/document library/Annual report/2017/05/ WC500227334.pdf (26. 11. 2017).

11. https://ec.europa.eu/research/fp7/index en.cfm (26. 11. 2017).

12. https://ec.europa.eu/programmes/horizon2020/ (26. 11. 2017).

13. Liua BC., Heb L., Hea G., Hee Y.: J. Public Health Policy 31, 407 (2010).

14. Vella Bonanno P., Ermisch M., Godman B., Antony P., Jesper Van Den Bergh M. et al.: Front. Pharmacol. 8, 497 (2017).

15. Regulation (EC) No $141 / 2000$ of the European Parliament and of the Council of 16 December 1999 on orphan medicinal products, Official Journal of the European Communities http://eur-lex.europa.eu/LexUriServ/Lex-
UriServ.do?uri=OJ:L:2000:018:0001:0005:en: PDF (26. 11. 2017).

16. http://www.ema.europa.eu/ema/index.jsp? curl=pages/regulation/general/general content 000029.jsp (26. 11. 2017).

17. http://www.ema.europa.eu/ema/index.jsp? curl=pages/about us/general/general content 000263.jsp (26. 11. 2017).

18. http://www.ema.europa.eu/ema/index.jsp? curl=pages/regulation/q and a/q and a detail 000014.jsp\&mid=WC0b01ac058061ecb9 (26. 11. 2017).

19. http://www.ema.europa.eu/ema/index.jsp? curl=pages/regulation/general/general content 000392.jsp (01. 12. 2017).

20. Regulation (EC) No 1901/2006 of the European Parliament and of the Council of 12 December 2006 on medicinal products for paediatric use and amending Regulation (EEC) No 1768/92, Directive 2001/20/EC, Directive 2001/83/EC and Regulation (EC) No 726/2004, Official Journal of the European Union (2006).

21. http://www.ema.europa.eu/ema/index.jsp? curl=pages/regulation/general/general content 000607.jsp\&mid=WC0b01ac0580925b1c (03. 12. 2017).

22. http://www.ema.europa.eu/ema/index.jsp? curl=pages/regulation/general/general content 000393.jsp (03. 12. 2017).

23. Regulation (EC) No 726/2004 of the European Parliament and of the Council of 31 March 2004 laying down Community procedures for the authorisation and supervision of medicinal products for human and veterinary use and establishing a European Medicines Agency, Official Journal of the European Union.

24. European Commission: COMMISSION REGULATION (EC) No 2049/2005 of 15 December 2005 laying down, pursuant to Regulation (EC) No 726/2004 of the European Parliament and of the Council, rules regarding the payment of fees to, and the receipt of administrative assistance from, the European Medicines Agency by micro, small and medium-sized enterprises, L 329/4-7, Official Journal of the European Union (2005).

25. http://www.ema.europa.eu/ema/index.jsp? curl=pages/regulation/general/general content 000519.jsp (03. 12. 2017).

26. European Medicines Agency, Committee for Medicinal Products for Human Use (CHMP): Guideline on the Procedure for Accelerated Assessment Pursuant to Article 14 (9) of Regulation (EC) No 726/2004, London, 17 July 2006 Doc. Ref. EMEA/419127/05. 
27. http://www.ema.europa.eu/ema/index.jsp? curl=pages/regulation/q and $\mathrm{a} / \mathrm{q}$ and a detail 000014.jsp\&mid=WC0b01ac058061ecb 9 (03. 12. 2017).

28. http://www.ema.europa.eu/ema/index.jsp? curl=pages/regulation/general/general content 000925.jsp (03. 12. 2017).

29. Directive 2001/83/EC of the European Parliament and of the CouncilOF THE EUROPEAN PARLIAMENT AND OF THE COUNCIL of 6 November 2001 on the Community code relating to medicinal products for human use, part II. 6, (OJ L 311, 28.11.2001, p. 67).

30. http://www.ema.europa.eu/ema/index.jsp? curl=pages/regulation/general/general content 000660.jsp (03. 12. 2017).

31. http://www.ema.europa.eu/docs/en GB/document library/Leaflet/2016/03/WC500202670. pdf (03. 12. 2017).

32. http://www.ema.europa.eu/ema/pages/includes/ document/open document.jsp?webContentId= WC500214862 (03. 12. 2017).

33. http://www.ema.europa.eu/ema/index.jsp? curl=pages/regulation/general/general content 000293.jsp (03. 12. 2017).

34. Balasubramanian G., Morampudi S., Chhabra P., Gowda A., Zomorodi B.: Intractable Rare Dis. Res. 5, 244 (2016).

35. http://www.aifa.gov.it/content/legge-64896 (03. 12. 2017).

36. Text of the Decree-Law of 20 March 2014, n. 36 (in the Official Gazette - general series - No. 67 of 21 March 2014), coordinated with the conversion law of 16 May 2014, no. 79 (in this Official Gazette on page 1), containing: «Urgent provisions concerning the regulation of narcotic drugs and psychotropic substances, prevention, treatment and rehabilitation of the related states of drug addiction, as per the Presidential Decree of 9 October 1990 , n. 309, as well as the use of medicines. " (14A03883) (GU General Series n.115 of 20-05-2014) http:/www.gazzettaufficiale.it/atto/serie generale/caricaDettaglioAtto/ originario?atto.dataPubblicazioneGazzetta $=2014-05-20 \&$ atto. codiceRedazionale $=$ 14A03883\&elenco30giorni=true (03. 12. 2017).

37. Weda M., Hoebert J., Vervloet M., Moltó Puigmarti C., Damen N. et al.: Study on offlabel use of medicinal products in the European Union (2017) https://ec.europa.eu/health/sites/ health/files/files/documents/2017 0228 final study report on off-label use.pdf (03. 12. 2017).

38. ANSM List of pharmaceutical products subject to an RTU - ANSM Liste des spécialités faisant l'objet d'une RTU. http://ansm.sante.fr/Activites/Recommandations-Temporaires-d-Utilisation-RTU/Liste-des-specialites-faisantactuellement-l-objet-d-une-RTU/(offset)/1.

39. The French Agency for the Safety of Health Products/Agence Nationale de Sécurité du Médicament et des produits de santé: Temporary Recommendation for Use (RTUs) Principles and information on the methods used by the ANSM for establishment and implementation October 2012 ansm.sante.fr/content/download/45542/590551/version/1/file/RTU-english.pdf (03. 12. 2017).

40. Social Security Financing Act No 2012-1404 for 2013 of the 17th December 2012. Official Journal of the French Republic of the $18^{\text {th }}$ December 2012, LOI no 2012-1404 du 17 décembre 2012 de financement de la sécurité sociale pour 2013, JORF no 0294 du 18 décembre 2012 page 19821texte no 2 https:/www. legifrance.gouv.fr/affichTexte.do?cidTexte $=\mathrm{JO}$ RFTEXT000026785322\&categorieLien $=i d$ (03. 12. 2017).

41. France Code of Public Health - Article L512112-1, Amended Social Security Financing Act No 2014-892 for 2014 of the $8^{\text {th }}$ August 2014 https://www.legifrance.gouv.fr/affichCodeArtic le.do?cidTexte=LEGITEXT000006072665\&id Article=LEGIARTI000025086118 (03.12.2017).

42. Avvocati Associati: Dal Negro Setti F., Cappellaro E.: Italy approves a new regulation on compassionate use of medicinal products (2017) https://www.lexology.com/library/ detail.aspx ?g=3c91ca00-7377-47cb-b0f6455f2195e129 (03.04.2017).

43. Italian Parliament: Law 8 April 1998, n. 94 "Conversion into law, with amendments, of the Decree-Law of 17 February 1998, No. 23, containing urgent provisions concerning clinical trials in the field of oncology and other measures in the field of health" published in the Official Gazette no. 86 of 14 April 1998 http://www.camera.it/parlam/leggi/980941.htm (03. 12. 2017).

44. http://www.ema.europa.eu/ema/index.jsp? curl=pages/regulation/general/general content 001857.jsp\&mid=WC0b01ac0580a11c96 (03. 12. 2017).

45. http://www.ema.europa.eu/ema/index.jsp? curl=pages/news and events/news/2017/11/ news detail 002850.jsp\&mid=WC0b01 ac058004d5c1\# (03. 12. 2017).

46. http://eunethta.eu/contactus/all/356/all (03.12. 2017). 
47. http://www.eunethta.eu/activities (03.12.2017).

48. https://www.eurordis.org/content/moca (03. 12. 2017).

49. https://ec.europa.eu/health/documents/pharmaceutical-committee/stamp en (03.12.2017).

50. European Commission Health and Food Safety Directorate-General, Report on activity of the Expert Group on Safe and Timely Access to Medicine for Patients (STAMP) 2015 - 2016, PHARM 728 REPORT https://ec.europa. eu/ health/sites/ health/files/files/committee/78meeting/pharm728 2ii stamp activity report final.pdf (03.12.2017).

51. EFPIA: The Innovative Medicines Initiatives, Key facts\&figures, September 2015 https: //www. imi.europa.eu/sites/default/files/ uploads/documents/reference-documents/Facts AndFigures Sept2015.pdf (03. 12. 2017).

52. http://www.imi.europa.eu/about-imi/historyimi-story-so-far (03. 12. 2017).

53. http://www.imi.europa.eu/about-imi/strategicresearch-agenda (03. 12. 2017).

54. http://www.imi.europa.eu/about-imi/missionobjectives (03. 12. 2017).

55. Innovative Medicine Initiative, EFPIA, European Union Horizon 2020 Research: ADAPTSMART, Innovative Medicine Discussion paper on Engagement Criteria for MAPPs (2016) http://adaptsmart.eu/wp-content/uploads/2016/ 02/ADAPT-SMART-Engagement-CriteriaFinal1.pdf (03. 12. 2017).

56. www.adaptsmart.eu (03.12.2017).

57. http://cordis.europa.eu/result/rcn/51670 en.html (03. 12. 2017).

58. Fresco LO., Martinuzzi A., Wiman A. et al.: Commitment and Coherence - Ex-Post Evaluation of the $7^{\text {th }}$ EU Framework Programme (2015).

59. https://ec.europa.eu/research/fp7/index en.cfm (03.12.2017).

60. http://cordis.europa.eu/programme/rcn/18551 en.html (03.12.2017).

61. EvaluatePharma: Orphan Drug Report 2015, 3rd Edition (2015) http://info.evaluategroup. com/rs/607-YGS-364/images/EPOD15.pdf (03. 12. 2017).

62. http://cordis.europa.eu/result/rcn/203187 en.html (03. 12. 2017).

63. http://cordis.europa.eu/project/rcn/110064 en.html (03. 12. 2017).

64. http://cordis.europa.eu/result/rcn/176580 en.html (03. 12. 2017).

65. Stallard N.: InSPiRe: Innovative methodology for small populations research An overview of research, Warwick Medical School, The Univer- sity of Warwick, UK http://www.ema. europa.eu/docs/en GB/document library/Presentation/2017/04/WC500226102.pdf (03. 12. 2017).

66. van der Lee H., Gaasterland C., Urach S.: Goal Attainment Scaling, an individualized instrument with potential for outcome measurement in rare diseases http://www.ema.europa.eu/ docs/en GB/document library/Presentation/ 2017/04/WC500226119.pdf.

67. Kiresuk T.J., Sherman R.E.: Community Ment. Health J. 4, 443 (1968).

68. http://www.ema.europa.eu/ema/index.jsp? curl=pages/news and events/events/2017/02/ event detail 001393.jsp\&mid=WC0b01ac058004d5c3 (03. 12. 2017).

69. An Act, To amend the Federal Food, Drug, and Cosmetic Act to facilitate the development of Jan. 411983 drugs for rare diseases and conditions, and for other purposes. Be it enacted by the Senate and House of Representatives of the United States of America in Congress assembled, Public Law, 97-414-JAN. 4, (1983) https://www.fda.gov/downloads/ForIndustry/D evelopingProductsforRareDiseasesConditions/ HowtoapplyforOrphanProductDesignation/UC M517741.pdf (dated 03. 12. 2017).

70. U.S. Food and Drug Administration Center for Drug Evaluation and Research, Novel Drugs Summary (2016)

71. https://www.fda.gov/Drugs/\% 20DevelopmentApprovalProcess/DrugInnovation/ucm474696. htm (03. 12. 2017).

72. McConaghie A., Europe launches new PRIME fast-track for promising medicines, Pharmaphorum, (2016) https://pharmaphorum.com/ articles/europe-launches-new-prime-fast-trackfor-promising-medicines/ (03. 12. 2017).

73. https://ec.europa.eu/health/sites/health/files/technology_assessment/docs/com2018_51_en.pdf (01. 07. 2018).

74. Lanthier M.: Economics Staff FDA Office of Planning Office of the Commissioner: Expedited Programs, Development Milestones, and the Benefits of FDA Engagement, NORD US Conference on Rare Diseases \& Orphan Products (2014) http://www.cbinet.com/sites/ default/files/files/Summit1 Lanthier.pdf (03. 12. 2017).

75. https://www.eurordis.org/sites/default/files/ publications/Fact Sheet RD.pdf (03. 12. 2017).

Received: 03.04.2018 